\title{
Nomenclature and semantic descriptions of ulcerative and inflammatory lesions seen in Crohn's disease in small bowel capsule endoscopy: An international Delphi consensus statement
}

United European Gastroenterology Journal 2020, Vol. 8(1) 99-107 (C) Author(s) 2020 Article reuse guidelines: sagepub.com/journals-permissions DOI: $10.1177 / 2050640619895864$ journals.sagepub.com/home/ueg @SAGE

\author{
Romain Leenhardt ${ }^{1}$, Anthony Buisson ${ }^{2}$, Arnaud Bourreille ${ }^{3}$, \\ Philippe Marteau ${ }^{1}$, Anastasios Koulaouzidis ${ }^{4}$, Cynthia Li $^{1,5}$, Martin Keuchel ${ }^{6}$, \\ Emmanuele Rondonotti ${ }^{7}$, Ervin Toth $^{8}$, John N Plevris ${ }^{4}$, Rami Eliakim? \\ Bruno Rosa ${ }^{10}$, Konstantinos Triantafyllou ${ }^{11}$, Luca Elli ${ }^{12}$, \\ Gabriele Wurm Johansson ${ }^{8}$, Simon Panter ${ }^{13}$, Pierre Ellul ${ }^{14}$, \\ Enrique Pérez-Cuadrado Robles ${ }^{15,25}$ (D), Deirdre McNamara ${ }^{16}$, \\ Hanneke Beaumont ${ }^{17}$, Cristiano Spada ${ }^{18}$, Flaminia Cavallaro ${ }^{19}$, \\ Franck Cholet $^{20}$, Ignacio Fernandez-Urien Sainz ${ }^{21}$, Uri Kopylov ${ }^{9}$, \\ Mark E McAlindon ${ }^{22}$, Artur Németh ${ }^{8}$, Gian Eugenio Tontini ${ }^{23}$, Diana E Yung ${ }^{4}$, \\ Yaron $\mathrm{Niv}^{24}$, Gabriel Rahmi ${ }^{25}$, Jean-Christophe Saurin ${ }^{26}$ and Xavier Dray ${ }^{1}$
}

Abstract

Background: In the medical literature, the nomenclature and descriptions (ND) of small bowel (SB) ulcerative and inflammatory (U-I) lesions in capsule endoscopy (CE) are scarce and inconsistent. Inter-observer variability in interpreting these

\footnotetext{
${ }^{1}$ Sorbonne Université, Endoscopy Unit, Hôpital Saint-Antoine, AP-HP, Paris, France

${ }^{2}$ Dept. of Gastroenterology, CHU Estaing Clermont-Ferrand, Clermont-ferrand, France

${ }^{3}$ Institut des Maladies de l'Appareil Digestif (IMAD), Dept of Gastroenterology, CHU Nantes, University of Nantes, Nantes, France

${ }^{4}$ Centre For Liver \& Digestive Disorders, The Royal Infirmary of Edinburgh, Edinburgh, United Kingdom

${ }^{5}$ College of Arts \& Sciences, Drexel University, Philadelphia, USA

${ }^{6}$ Klinik für Innere Medizin, Bethesda Krankenhaus Bergedorf, Hamburg, Germany

${ }^{7}$ Gastroenterology Unit, Valduce Hospital, Como, Italy

${ }^{8}$ Department of Gastroenterology, Skåne University Hospital, Lund University, Malmö, Sweden

${ }^{9}$ Dept. of Gastroenterology, Sheba Medical Center, Ramat Gan, and Sackler School of Medicine, Tel-Aviv University, Tel-Aviv, Israel

${ }^{10}$ Departamento de Gastroenterologia, Universidade do Minho, Hospital Senhora da Oliveira, Guimarães, Portugal

${ }^{11}$ Hepatogastroenterology Unit, Second Department of Internal Medicine Propaedeutic Research Institute and Diabetes Center, National and Kapodistrian University of Athens, Medical School, Attikon University General Hospital, Athens, Greece

${ }^{12}$ Center for Prevention and Diagnosis of Celiac Disease, Fondazione IRCCS Ca Granda, Milano, Italy

${ }^{13}$ Gastroenterology, South Tyneside Hospital, South Shields, United Kingdom

${ }^{14}$ Department of Medicine, Mater Dei Hospital, Msida, Malta
}

B5 Department of Gastroenterology, Cliniques Universitaires Saint-Luc,
Bruxelles, Belgium
${ }^{16}$ Trinity Academic Gastroenterology Group, Departement of Clinical
Medicine, Tallaght Hospital, Trinity College Dublin, Ireland
${ }^{17}$ Department of Gastroenterology, Amsterdam UMC, Vrije Universiteit
Amsterdam, Amsterdam, The Netherlands
${ }^{18}$ Digestive Endoscopy Unit and Gastroenterology, Fondazione
Poliambulanza, Brescia, Italia; Digestive Endoscopy Unit, Università
Cattolica del Sacro Cuore, Roma, Italia
${ }^{19}$ Gastroenterology and Endoscopy Unit, IRCCS Policlinico San Donato, San
Donato Milanese, Milan, Italy
${ }^{20}$ Endoscopy unit, CHU La Cavale Blanche, Brest, France
${ }^{21}$ Gastroenterology, Hospital de Navarra, Pamplona, Spain
${ }^{22}$ Dept. of Gastroenterology, Royal Hallamshire Hospital, Sheffield, United
Kingdom
${ }^{23}$ Department of Pathophysiology and Transplantation, University of Milan-
Gastroenterology and Endoscopy Unit, Fondazione IRCCS Ca' Granda
Ospedale Maggiore Policlinico, Milan, Italy
${ }^{24}$ Rabin Medical Center, Dept. of Gastroenterology, Petach Tikva, Israel, and
Sackler School of Medicine, Tel-Aviv University, Tel-Aviv, Israel
${ }^{25}$ Department of Gastroenterology and Digestive Endoscopy, Georges-
Pompidou European hospital, Paris, France
${ }^{26}$ Gastroenterology and Endoscopy Unit, Edouard Herriot Hospital, Lyon,
France Corresponding author:

Xavier Dray, Hôpital Saint-Antoine, 184 rue du Faubourg Saint-Antoine Paris, 75012 France.

Email: xavier.dray@aphp.fr 
findings remains a major limitation in the assessment of the severity of mucosal lesions, which can impact negatively on clinical care, training and research on SB-CE.

Objective: Focusing on SB-CE in Crohn's disease (CD), our aim is to establish a consensus on the ND of U-I lesions.

Methods: An international panel of experienced SB-CE readers was formed during the 2016 United European Gastroenterology Week meeting. A core group of five CE and inflammatory bowel disease (IBD) experts established an Internet-based, three-round Delphi consensus but did not participate in the voting process. The core group built illustrated questionnaires, including SB-CE still frames of U-I lesions from patients with documented CD. Twenty-seven other experts were asked to rate and comment on the different proposals for the ND of the most frequent SB U-I lesions. For each round, we used a 6-point rating scale (varying from 'strongly disagree' to 'strongly agree'). The consensus was reached when at least $80 \%$ of the voting members scored the statement within the 'agree' or 'strongly agree' categories.

Results: A $100 \%$ participation rate was obtained for all the rounds. Consensual ND were reached for the following seven U-I lesions: aphthoid erosion, deep ulceration, superficial ulceration, stenosis, edema, hyperemia and denudation.

Conclusion: Considering the most frequent SB U-I lesions seen in CE in CD, a consensual ND was reached by the international group of experts. These descriptions and names are useful not only for daily practice and medical education, but also for medical research.

\section{Keywords}

Capsule endoscopy, small-bowel, Crohn's disease, Delphi consensus, inflammatory bowel diseases, nomenclature

Received: 31 August 2019; accepted: 24 November 2019

\section{Introduction}

Considering its high ability to visualise the entire small bowel (SB) mucosa, capsule endoscopy (CE) has rapidly been considered as one of the first-line diagnostic tools for $\mathrm{SB}$ examination. ${ }^{1} \mathrm{CE}$ has an approximate diagnostic yield of $50 \%$ in suspected Crohn's disease (CD) cases $^{2}$ and plays a significant role in its management. ${ }^{3,4}$ The Capsule Endoscopy Crohn's Disease Activity Index (CECDAI) was developed ${ }^{5}$ and validated ${ }^{6}$ to grade the severity of SB-CE findings in patients with $\mathrm{CD}$. The Lewis score also provides a common language to quantify mucosal inflammatory variations. ${ }^{7}$ Both scores are commonly used both in clinical practice and in research because they display a strong correlation. ${ }^{8}$ However, the nomenclature and descriptions (ND) of ulcerative and inflammatory (U-I) lesions seen in CE and that are used in these scores are far from established. For instance, in the study by Gralnek et al., the 'definitions of endoscopic lesions were a priori specified and reviewed by all readers prior to prospective testing', yet no definitions were consensually agreed and provided. ${ }^{7}$ As CE reviewing is strongly related to the reader's opinion and experience, which differ among readers, ${ }^{9,10}$ it is crucial to search for a common international ND to define the most common U-I lesions seen on SB-CE in patients with CD.

Indeed, in the medical literature, studies assessing the inter-observer variability have measured a mean kappa value of around $0.5 .{ }^{11} \mathrm{We}$ believe that the inter-observer variability in interpreting the findings may be (at least partly) due to the lack of a common language, and that it is a major limitation for assessing the severity of mucosal lesions observed in inflammatory bowel diseases (IBD), with a potential negative impact on patients' healthcare and on the evaluation of therapeutics. Therefore, it seems necessary to standardise and use a unified language for all major U-I lesions seen during SB-CE in IBD. A similar approach has been used by our group through the establishment of an international consensus on the ND of the most frequent vascular lesions seen in SB-CE. ${ }^{12}$ Hence, the aim of the current study was to establish a consensual ND of the most common U-I lesions seen on SB-CE in patients with CD.

\section{Patients, materials and methods \\ Design}

The Delphi method is one of the most accurate and validated process for answering questions that cause uncertainty even among experts. ${ }^{13,14}$ The present study used a Delphi consensus model based on alternative feedback rounds between a core group (CG) and an expert group (EG) until a consensus was reached (Figure 1). The CG built several Internet-based illustrated questionnaires and asked the EG to rate the descriptions and add comments to inform the next round. The questionnaires were structured similarly, presenting images related to several propositions for the nomenclature and the semantic descriptions of U-I lesions seen in CD patients. All members of the EG were invited to add comments for each proposal. Based on the rating and on the comments received from the 


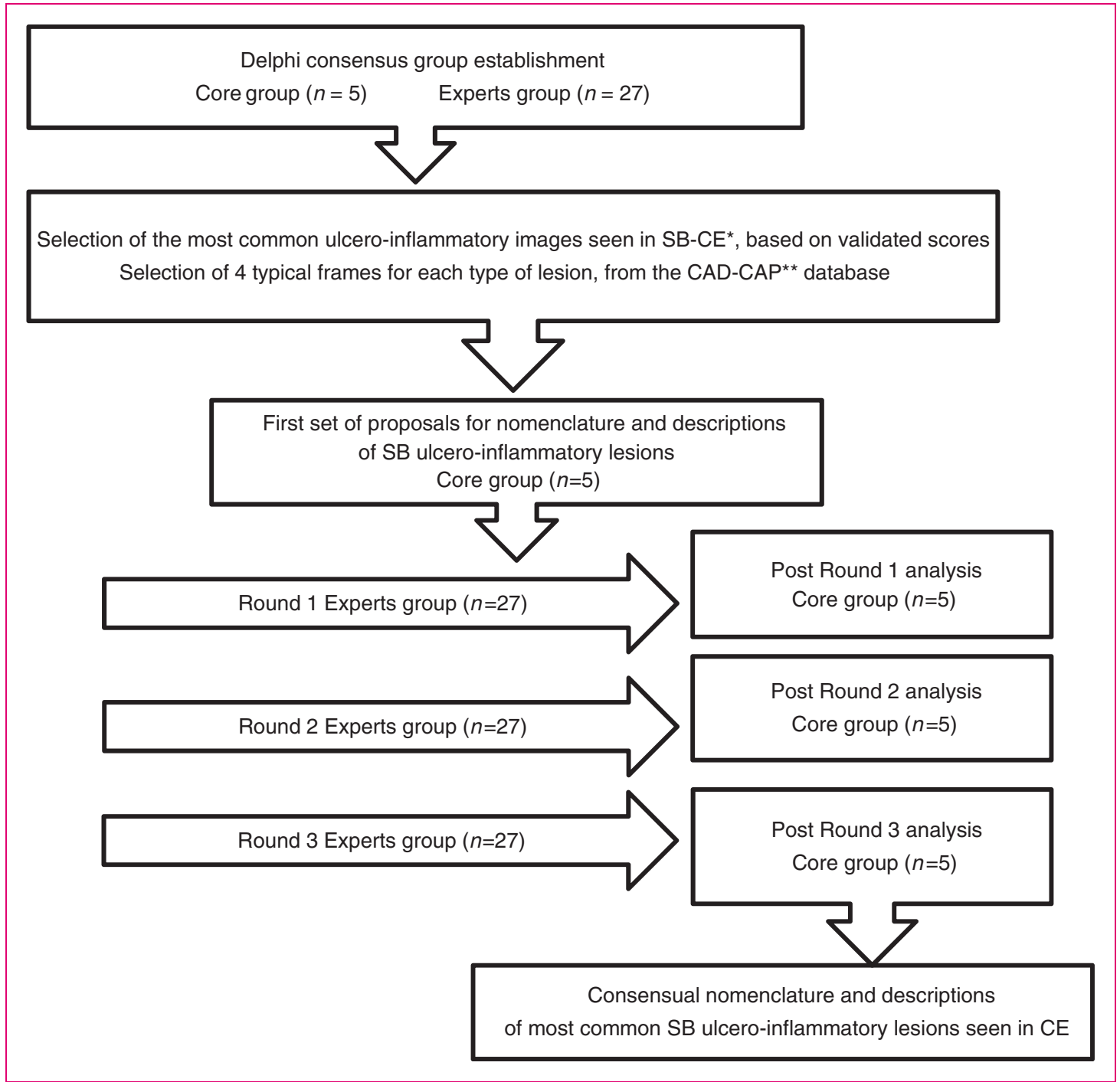

Figure 1. Delphi consensus study flowchart. ${ }^{\star S B}-$ CE: small bowel capsule endoscopy. ${ }^{\star \star C A D-C A P: ~ C o m p u t e r-a i d e d ~ D i a g n o s i s ~ f o r ~ c a p s u l e ~}$ endoscopy database (19).

experts, the name and the description of each lesion were then reviewed by the $\mathrm{CG}$ after each feedback round.

\section{Working groups and feedback rounds}

The working groups were constituted during the 24th United European Gastroenterology Week (15-19 October 2016, Vienna, Austria). The CG was composed of five French IBD specialists, with three of them also being SB-CE expert readers. The CG members did not participate in the EG. The 27 members of the international EG were based in England $(n=2)$, France $(n=3)$, Germany $(n=1)$, Greece $(n=1)$, Ireland $(n=1)$, Israel $(n=3)$, Italy $(n=5)$, Malta $(n=1)$, the Netherlands $(n=1)$, Portugal $(n=1)$, Scotland $(n=3)$, Spain $(n=2)$ and Sweden $(n=3)$. The mean age of the EG members was 47.6 years.
The mean CE reading experience of the EG members was $12.4 \pm 4.6$ years, with a mean number of $136 \pm 85$ CEs read annually.

\section{Proposals and revisions of nomenclature and semantic descriptions}

To design the first round, the CG first reviewed relevant U-I terms described in the SB-CE IBD scores ${ }^{5,7}$ or in the papers of the digestive endoscopic terminology. ${ }^{15-17}$ Moreover, to avoid confusion, the CG also considered the terms and definitions of U-I lesions found during colonoscopy of IBD patients, as proposed by Groupe d'Etudes Thérapeutiques des Affections Inflammatoires du Tube Digestif (GETAID). ${ }^{18}$

The CG proposed a selection of four typical SB-CE image frames, a nomenclature and a semantic 
description per specific type of SB U-I lesion. All selected frames were extracted from the multicentre French database CAD-CAP (computer-assisted diagnosis for capsule endoscopy). This database is composed of 20,000 normal CE still frames and 5184 stillframe images of abnormal $\mathrm{CE}$ findings from third-generation SB-CE system videos (Pillcam ${ }^{\circledR} \mathrm{SB} 3$ system, Medtronic), including 1369 images with U-I lesions from patients with IBD. ${ }^{19}$ It has already been used to standardise and illustrate a common lexicon for vascular lesions seen in SB-CE ${ }^{12}$ and to develop CAD tools for capsule reading. ${ }^{20}$ An Internet-based survey was used to send all the propositions to the EG.

For each round, the EG members were invited to rate the propositions made for each type of lesion, using a numerical scale varying from 1 (strongly disagree) to 6 (strongly agree) (Table 1). They were also asked to provide comments and suggestions for subsequent revisions. To improve the lesions ND, the CG reviewed the EG's suggestions after each round.

\section{Predefined stop criterion and analysis}

We considered a consensual agreement was reached when over $80 \%$ of the voting members rated with a score of 5 (agree) or 6 (strongly agree) within three rounds. The Delphi consensus process was stopped when the consensual name and description of any lesion was established. Meetings were organised between the five participants of the $C G$ within the first three rounds to discuss the votes and comments. Subsequently, whether a consensus was reached or not, the process was interrupted.

\section{Results}

The CG identified the seven following lesions to be proposed to the EG for definition and description: aphthoid erosion, deep ulceration, superficial ulceration, stenosis, edema, denudation and hyperemia. A $100 \%$ participation rate was obtained from the EG for all three rounds. The consensual ND, as finally obtained at the end of the Delphi process, are given in Table 2. The experts' ratings are detailed in Tables 3 and 4 for the nomenclature and descriptions, respectively.

\section{Aphthoid erosion}

Regarding Figure 2(a), the nomenclature voted on for this type of lesion after one round with an $85.2 \%$ agreement rate was 'aphthoid erosion'. After round 2, the description 'Diminutive loss of epithelial layering with a whitish centre and a red halo, surrounded by normal mucosa' obtained a $96.3 \%$ agreement rate.
Table 1. Six-point numerical scale to rate proposals during the Delphi rounds.

\begin{tabular}{ll}
\hline 1 & Strongly disagree \\
2 & Disagree \\
3 & Moderately disagree \\
4 & Moderately agree \\
5 & Agree \\
6 & Strongly agree \\
\hline
\end{tabular}

\section{Deep ulceration}

Regarding Figure 2(b), the nomenclature 'deep ulceration' was voted on for this type of lesion after one round with a $96.3 \%$ rate of agreement. The description 'Frankly, deep loss of tissue compared with the surrounding swollen/edematous mucosa, with a whitish base' reached an $85.2 \%$ rate of agreement after round 3 .

\section{Superficial ulceration}

Regarding Figure 2(c), the nomenclature 'superficial ulceration' was voted on for this type of lesion after one round with an $81.5 \%$ rate of agreement. The rate of agreement for the description 'Mildly depressed loss of tissue with a whitish bottom, whose features fit neither with that of aphthoid erosion nor with that of deep ulceration, as previously defined' was of $85.2 \%$ after round 2. Although referring to the descriptions of 'deep ulceration' and 'aphthoid erosion', a consensual description for 'superficial ulceration' was obtained before this, during round 2 .

\section{Stenosis}

Regarding Figure 3(a), after one round the nomenclature 'stenosis' was voted on for this type of lesion with a $100.0 \%$ rate of agreement. During round 1 the EG asked for videos to further discuss the description. A 9-second video sequence ( 25 frames upstream, and 25 downstream the index still frame) was added to the questionnaire during round 2, as well as a special mention for the need of a dynamic sequence to establish a diagnosis. After round 2, the rate of agreement of the following description: 'Narrowing of the intestinal lumen withholding or delaying the passing of the videocapsule (therefore, to be evaluated on a video)' was of $88.9 \%$.

\section{Edema}

After round 1, the EG asked for modifications in the selection of the SB-CE illustrations. Regarding 
Table 2. International Delphi consensus on the nomenclature and descriptions of ulcerative and inflammatory lesions in SB-CE.

\begin{tabular}{|c|c|c|}
\hline Nomenclature & Description & $\begin{array}{l}\text { \% Nomenclature/ } \\
\text { description }\end{array}$ \\
\hline Aphthoid erosion & $\begin{array}{l}\text { Diminutive loss of epithelial layering with a whitish center and a red halo, } \\
\text { surrounded by normal mucosa }\end{array}$ & $85.2 / 96.3$ \\
\hline Deep ulceration & $\begin{array}{l}\text { Frankly deep loss of tissue compared to the surrounding swollen/edematous } \\
\text { mucosa, with a whitish base }\end{array}$ & $96.3 / 85.2$ \\
\hline Superficial ulceration & $\begin{array}{l}\text { Mildly depressed loss of tissue with a whitish bottom, whose features fit neither } \\
\text { with that of aphthoid erosion nor with that of deep ulceration, as previously defined }\end{array}$ & $81.5 / 85.2$ \\
\hline Stenosis & $\begin{array}{l}\text { Narrowing of the intestinal lumen withholding or delaying the passing of the } \\
\text { videocapsule (therefore, to be evaluated on a video) }\end{array}$ & $100.0 / 88.9$ \\
\hline Edema & Enlarged / swollen / engorged villi & $85.2 / 81.5$ \\
\hline Hyperemia & Area of reddish villi & $96.3 / 81.5$ \\
\hline Denudation & Reddish (but not whitish) mucosal area where villi are absent & $81.5 / 81.5$ \\
\hline
\end{tabular}

Table 3. Experts' rating regarding the nomenclature of the selected ulcerative and inflammatory SB lesions.

\begin{tabular}{|c|c|c|c|c|c|c|c|c|}
\hline \multirow[b]{2}{*}{ Nomenclature } & \multicolumn{6}{|c|}{ Numerical scale / Number of expert voting } & \multirow{2}{*}{$\begin{array}{l}\text { \% of 'agree' or } \\
\text { 'strongly agree' }\end{array}$} & \multirow{2}{*}{$\begin{array}{l}\text { Number of } \\
\text { rounds passed }\end{array}$} \\
\hline & 1 & 2 & 3 & 4 & 5 & 6 & & \\
\hline Aphthoid erosion & 2 & - & 2 & - & 12 & 11 & 85.2 & 1 \\
\hline Deep ulceration & 1 & - & - & - & 5 & 21 & 96.3 & 1 \\
\hline Superficial ulceration & - & - & 2 & 3 & 11 & 11 & 81.5 & 1 \\
\hline Stenosis & - & - & - & - & 9 & 18 & 100.0 & 1 \\
\hline Edema & - & 1 & 1 & 2 & 12 & 11 & 85.2 & 2 \\
\hline Hyperemia & - & - & 1 & - & 21 & 6 & 96.3 & 3 \\
\hline Denudation & - & 1 & - & 4 & 10 & 12 & 81.5 & 1 \\
\hline
\end{tabular}

Table 4. Experts' rating on the description of the selected ulcerative and inflammatory SB lesions.

\begin{tabular}{|c|c|c|c|c|c|c|c|c|}
\hline \multirow[b]{2}{*}{ Description } & \multicolumn{6}{|c|}{ Numerical scale / Number of expert voting } & \multirow{2}{*}{$\begin{array}{l}\% \text { of 'agree' or } \\
\text { 'strongly agree' }\end{array}$} & \multirow{2}{*}{$\begin{array}{l}\text { Number of } \\
\text { rounds passed }\end{array}$} \\
\hline & 1 & 2 & 3 & 4 & 5 & 6 & & \\
\hline Aphthoid erosion & - & - & - & 1 & 13 & 13 & 96.3 & 2 \\
\hline Deep ulceration & - & 1 & - & 3 & 14 & 9 & 85.2 & 3 \\
\hline Superficial ulceration & - & - & - & 4 & 15 & 8 & 85.2 & 2 \\
\hline Stenosis & - & - & 1 & 2 & 13 & 11 & 88.9 & 2 \\
\hline Edema & - & 1 & - & 4 & 14 & 8 & 81.5 & 2 \\
\hline Hyperemia & & 1 & 1 & 3 & 14 & 8 & 81.5 & 3 \\
\hline Denudation & - & 1 & - & 4 & 10 & 12 & 81.5 & 1 \\
\hline
\end{tabular}

Figure 3(b), the nomenclature voted on for this type of lesion after two rounds was an 'edema' with an $85.2 \%$ rate of agreement. A rate of agreement of $81.5 \%$ was reached after round 2 for the following description: 'Enlarged/swollen/engorged villi'.

\section{Hyperemia}

A hyperemia is a nonspecific finding often associated with other common U-I lesions. During the first two rounds the EG asked the $\mathrm{CG}$ for modifications in the selection of the SB-CE images. Thus, two frames were 


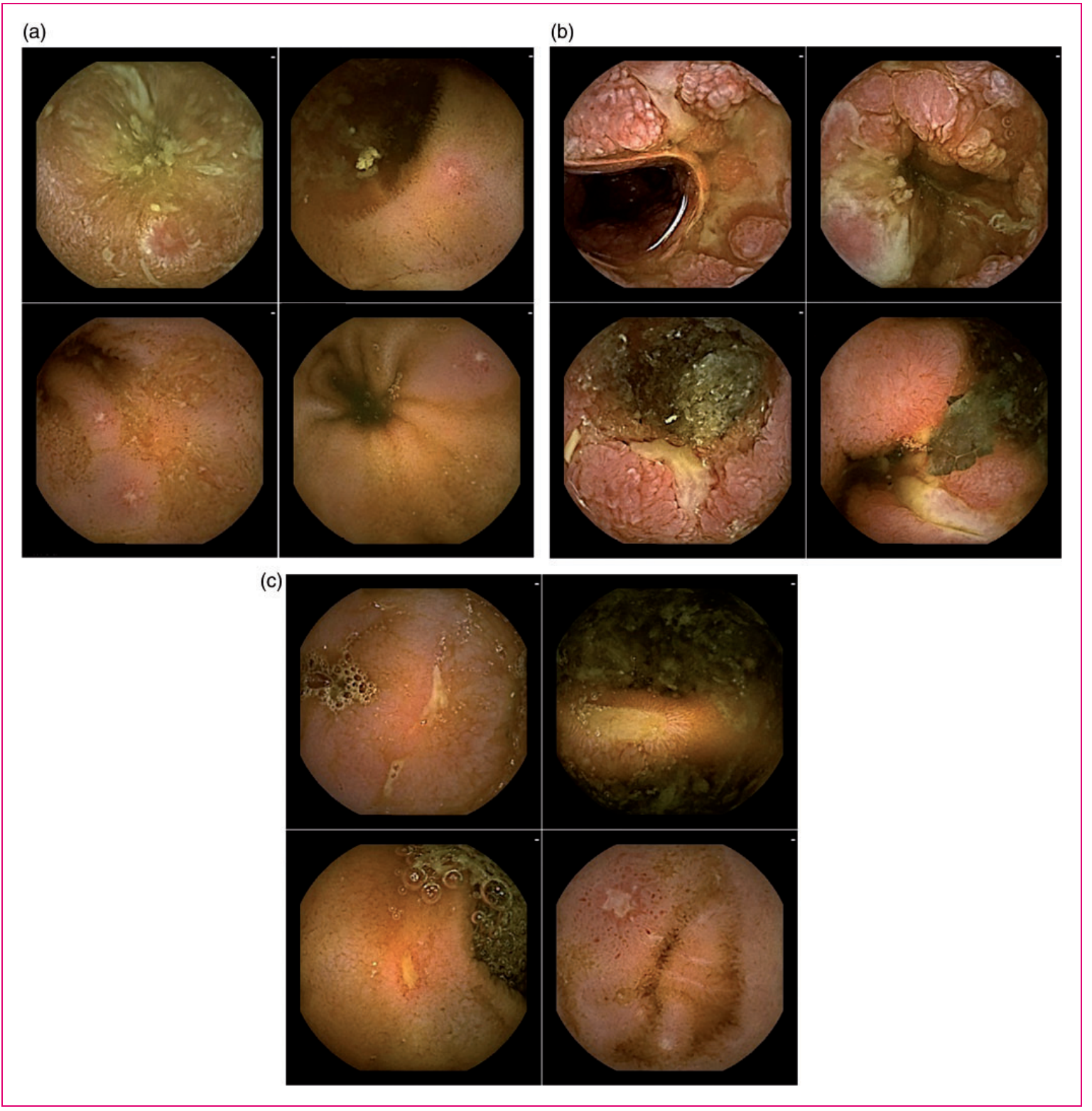

Figure 2. Frames with various types of loss of tissue. (a) Four typical frames of 'Aphthoid erosion'; (b) Four typical frames of 'Deep ulceration'; (c) Four typical frames of 'Superficial ulceration'.

removed from the initial selection. The regions of interest within the two remaining frames were delimited (Figure 3(d)). The nomenclature voted on for Figure 3(d) after three rounds was a 'hyperemia' with a $96.3 \%$ rate of agreement. An $81.5 \%$ rate of agreement was reached after round 3 for the description: 'Area of reddish villi'.

\section{Denudation}

Regarding Figure 3(c), an 81.5\% rate of agreement was reached for the nomenclature 'denudation' after one round. After round 1 the following description:
'Reddish (but not whitish) mucosal area where villi are absent' obtained an $81.5 \%$ rate of agreement.

\section{Discussion}

In the current study, the ND of seven common U-I lesions seen in SB-CE in IBD patients were validated by experts who reached a more than $80 \%$ consensus rate. The agreements were reached using a Delphi consensus process conducted with 27 expert CE readers.

Since its clinical inception in 2000, CE has revolutionised the examination and description of the SB. 


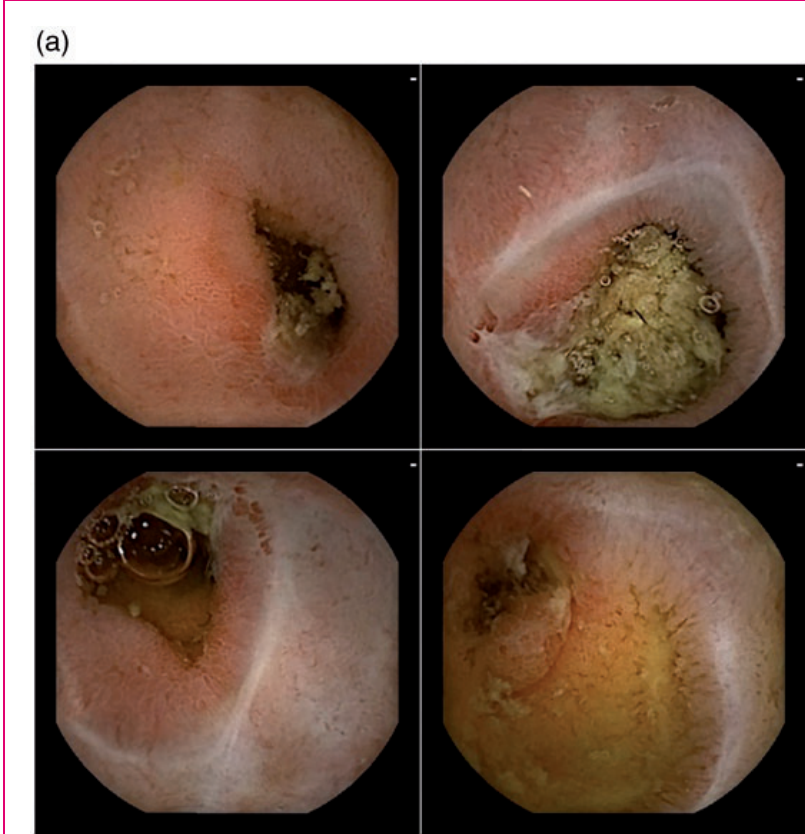

(c)

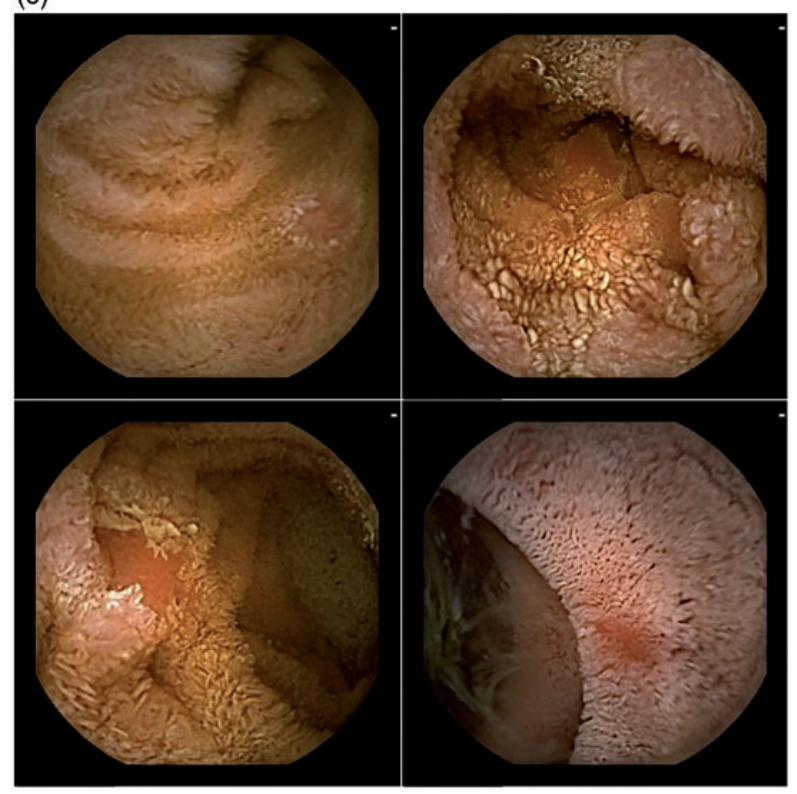

(b)

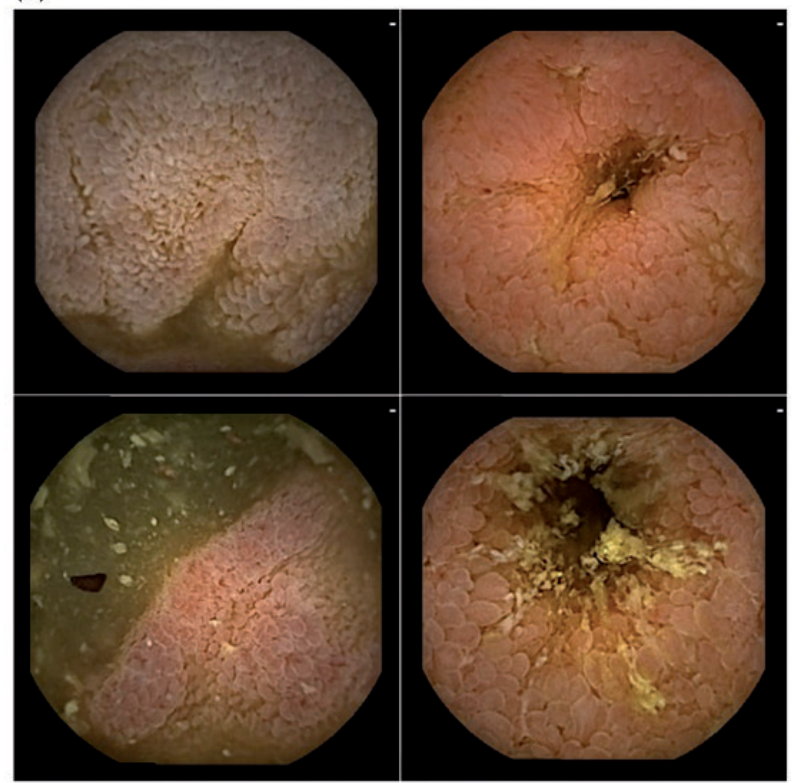

(d)

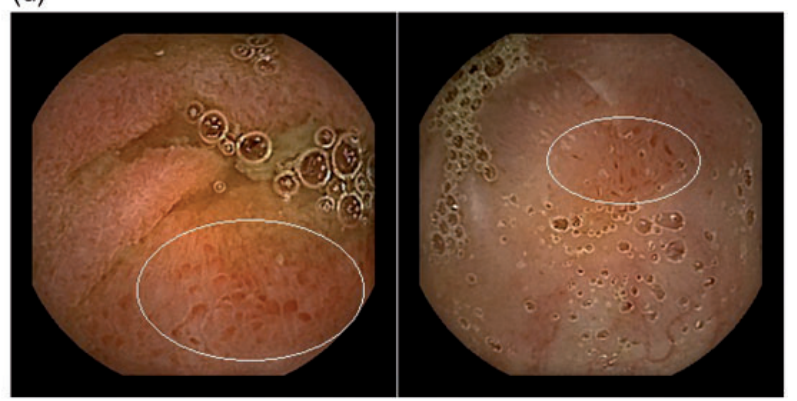

Figure 3. Frames with stenosis and with various types of inflammatory, non-ulcerated, lesions.

(a) Four typical frames of 'Stenosis'; (b) Four typical frames of 'Edema'; (c) Four typical frames of 'Denudation'; (d) Two typical frames of 'Hyperemia'.

Although a terminology index exists for $\mathrm{SB}-\mathrm{CE},{ }^{15}$ a consensual ND of SB findings has not been clearly established. This lack of ND makes it difficult to standardise SB-CE reading, reporting and research. Indeed, in most studies evaluating the inter-observer agreement in the description of SB-CE, only the experts' opinions are considered as the gold standard.

In an attempt to standardise the practice in SB-CE reading, our group first performed a Delphi consensus to better define and describe vascular lesions found in SB-CE. ${ }^{12}$ Similarly, we propose in the present study a consensus on the ND of U-I lesions seen in SB-CE in IBD patients. During the Delphi process, several important points were raised by the EG discussed subsequently by the CG. First, some U-I lesions are considered 'nonspecific', such as U-I lesions caused by the use of nonsteroidal anti-inflammatory drugs, by CD, by ischaemia or by other factors that cannot be easily or 
categorically distinguished. Second, in SB-CE, as there is currently no available tool to precisely measure lesions, we could only use adjectives of size (e.g. 'diminutive') to describe the lesions instead of standardised units of measurement. Third, several types of U-I lesions are often found together in the same SB-CE frame, making it difficult to define their specific descriptions and illustrations. As an example, a consensus on the term 'hyperemia' could not be achieved within the two first rounds as this finding is nonspecific and often associated with other common U-I lesions. However, this issue was solved by delineating the regions of interest within the selected frames. Fourth, it is important to emphasise the difficulties in clearly distinguishing a superficial from a deep ulceration. Indeed, the evaluation of the depth of loss of tissue remains subjective and variable between experts, and a villous appearance contiguous to a mucosal break should be considered to appreciate and better characterise the entire lesion.

The present study has many strengths. First, the questionnaires were richly illustrated with high-quality, third-generation SB-CE images to better guide the experts. When required by the EG, still frames could be changed or delineated, and short video sequences could be specifically linked to the still images (e.g. when commenting on the term 'stenosis'). Such complementary information was provided when needed to increase the precision in the lesion to be described and to ensure a high reliability in the votes. Second, a substantial number of experts $(n=27)$ voted, they had a significant experience (mean 12.4 years) and performed a high volume (mean 136 cases per year) of SB-CE readings. Third, a $100 \%$ participation rate was obtained in all three rounds. Fourth, all the answers were anonymous, which encouraged open critique, while avoiding and preventing some experts from dominating in the process. Fifth, based on the EG's answers and comments, the members of the CG wrote out proposals and revisions, but they did not participate in the vote.

Some limitations should be acknowledged. First, almost all of the investigators were based in Europe. However, the 27 EG members were from 13 different countries, thus offering some meaningful diversity for such a study. Conversely, the five members of the CG were all affiliated with France, where the project was initiated, but they belonged to three different institutions. Second, the selection of the seven types of lesions to be studied was made by the CG based on their experience and on a review of the literature that had a special focus on the items of the CECDAI and the Lewis score. ${ }^{5,7}$ The EG was not specifically asked whether any other type of U-I lesion should be added to the list and voted for. For the seven above-mentioned different types of lesions, however, the EG members could comment and ask for modifications. No EG expert requested adding an additional type of U-I to the list. Third, all images have been captured from the SB-CE Pillcam ${ }^{\circledR}$ SB3 system only. Therefore, it would be interesting to evaluate whether the ND described here are relevant with other SB-CE systems. Fourth, the current study represents a step forward in the standardisation of the description of $\mathrm{CE}$ lesions, and it does not assess the clinical relevance of such lesions according to specific clinical settings. Specifically, hyperemia and edema are subtle and debatable lesions. Their consensual descriptions by the experts were very short compared with that written for other lesions. These brief descriptions may indeed reflect the ideas of some IBD specialists that those findings may not be true manifestations or markers of CD, and that the validity of the Lewis Score is questionable for that reason..$^{21}$ Fifth, the current study aimed to precisely describe lesions, but there is currently no available measurement tool in SB-CE, and no possibility to sample the lesions. Descriptive terms with adjectives (e.g. 'whitish/yellowish bottom', 'narrowing') rather than objective measurements (e.g. diameter, depth in $\mathrm{mm} .$. ) or pathology terms (e.g. fibrin bottom...) were therefore used by both the CG and the EG. Timing was nevertheless available, and should have been quantified to precisely describe what the experts call 'withholding or delaying the passing of the videocapsule' when describing a stenosis. All in all, these descriptions may change when the SB-CE technology evolves and when readers call for even more precise and reproducible reports.

The proposed nomenclatures, atlas and descriptions of vascular and U-I lesions in SB-CE by our group pave to way to a study assessing their intra- and inter-observer reproducibility. We also plan to conduct survey on their clinical pertinence for the main indications of SB$\mathrm{CE}$ (occult or overt gastrointestinal bleeding, and suspicion of $\mathrm{CD}$ ).

\section{Conclusion}

An international group of SB-CE expert readers reached a consensus for the ND of the most frequent U-I lesions seen in CE. The current study has provided a strong asset for higher quality SB-CE reading and reporting in patients with $\mathrm{CD}$. These names and descriptions will also be useful for medical education and for medical research purposes.

\section{Declaration of conflicting interests}

The authors disclose the following:

Romain Leenhardt is cofounder and shareholder of Augmented Endoscopy, and has given lectures for Abbvie.

Xavier Dray is cofounder and shareholder of Augmented 
Endoscopy and has acted as a consultant for Alfasigma; Bouchara Recordati; Boston Scientific, Fujifilm, Medtronic, and Pentax.

Simon Panter has received support for a research fellow from Fuji and has acted as consultant for Medtronic.

Arnaud Bourreille has received consultant fees from Abbvie, Ferring, Janssen, Medtronic, MSD, Pfizer, Takeda, Tillotts, research grants from Abbvie, Takeda, Medtronic.

Uri Kopilov: has received consultant and speaker fees for Takeda, Abbvie, Jannsen, Medtronic. Research support from Jannsen, Medtronic, Takeda.

Rami Eliakim has received consultant fees from Abbvie, Takeda, Janssen and Medtronic; Research grant from Medtronic.

\section{Funding}

The Computer-Assisted Diagnosis for Capsule Endoscopy database has been made possible with the support of the Société Nationale Française de Gastroentérologie and with the support of MSD France.

\section{Ethics approval}

This Delphi consensus statement complies with the Declaration of Helsinki on research ethics.

\section{Informed consent}

De-identified images were extracted from the CAD-CAP database, a national multicenter database approved by the French Data Protection Authority.

\section{ORCID iD}

Enrique Pérez-Cuadrado Robles (D) https://orcid.org/00000001-8254-7453

\section{References}

1. Iddan G, Meron G, Glukhovsky A, et al. Wireless capsule endoscopy. Nature 2000; 405: 417.

2. Pennazio M, Spada C, Eliakim R, et al. Small-bowel capsule endoscopy and device-assisted enteroscopy for diagnosis and treatment of small-bowel disorders: European Society of Gastrointestinal Endoscopy (ESGE) Clinical Guideline. Endoscopy 2015; 47: 352-376.

3. Bourreille A, Ignjatovic A, Aabakken L, et al. Role of small-bowel endoscopy in the management of patients with inflammatory bowel disease: An international OMED-ECCO consensus. Endoscopy 2009; 41: 618-637.

4. Enns RA, Hookey L, Armstrong D, et al. Clinical practice guidelines for the use of video capsule endoscopy. Gastroenterology 2017; 152: 497-514.

5. Gal E, Geller A, Fraser G, et al. Assessment and validation of the new capsule endoscopy Crohn's disease activity index (CECDAI). Dig Dis Sci 2008; 53: 1933-1937.

6. Niv Y, Ilani S, Levi Z, et al. Validation of the Capsule Endoscopy Crohn's Disease Activity Index (CECDAI or Niv score): A multicenter prospective study. Endoscopy 2012; 44: 21-26.
7. Gralnek IM, Defranchis R, Seidman E, et al. Development of a capsule endoscopy scoring index for small bowel mucosal inflammatory change. Aliment Pharmacol Ther 2008; 27: 146-154.

8. Yablecovitch D, Lahat A, Neuman S, et al. The Lewis score or the capsule endoscopy Crohn's disease activity index: Which one is better for the assessment of small bowel inflammation in established Crohn's disease? Ther Adv Gastroenterol 2018; 11: 1756283X17747780.

9. Rondonotti E, Soncini M, Girelli CM, et al. Can we improve the detection rate and interobserver agreement in capsule endoscopy? Dig Liver Dis 2012; 44: 1006-1011.

10. Pezzoli A, Cannizzaro R, Pennazio $M$, et al. Interobserver agreement in describing video capsule endoscopy findings: A multicentre prospective study. Dig Liver Dis 2011; 43: 126-131.

11. Mergener K, Ponchon T, Gralnek I, et al. Literature review and recommendations for clinical application of small-bowel capsule endoscopy, based on a panel discussion by international experts. Endoscopy 2007; 39: 895-909.

12. Leenhardt R, Li C, Koulaouzidis A, et al. Nomenclature and semantic description of vascular lesions in small bowel capsule endoscopy: An international Delphi consensus statement. Endosc Int Open 2019; 7: E372-E379.

13. Dalkey $\mathrm{N}$ and Helmer $\mathrm{O}$. An experimental application of the DELPHI method to the use of experts. Manag Sci 1963; 9: 458-467.

14. Hsu C-C and Sandford BA. The Delphi technique: Making sense of consensus. Pract Assess Res Eval 2007; 12: $1-8$.

15. Korman LY, Delvaux M, Gay G, et al. Capsule endoscopy structured terminology (CEST): Proposal of a standardized and structured terminology for reporting capsule endoscopy procedures. Endoscopy 2005; 37: 951-959.

16. Aabakken L, Rembacken B, LeMoine O, et al. Minimal standard terminology for gastrointestinal endoscopyMST 3.0. Endoscopy 2009; 41: 727-728.

17. Delvaux M, Crespi M, Armengol-Miro JR, et al. Minimal standard terminology for digestive endoscopy: Results of prospective testing and validation in the GASTER project. Endoscopy 2000; 32: 345-355.

18. Buisson A, Filippi J, Amiot A, et al. Gastroenterology 2015;148,S-445-6 (abstract).

19. Leenhardt R, Li C, Le Mouel JP, et al. CAD-CAP: A 25000 images database serving the developement of artificial intelligence for capsule endoscopy. Endosc Int Open (in press).

20. Leenhardt R, Vasseur P, Li C, et al. A neural network algorithm for detection of GI angiectasia during smallbowel capsule endoscopy. Gastrointest Endosc 2019; 89: 189-194.

21. Omori T, Kambayashi H, Murasugi S, et al. Comparison of Lewis Score and Capsule Endoscopy Crohn's Disease Activity Index in patients with Crohn's disease. Dig Dis Sci2019 (in press). 